\title{
SERPINs in Macrophage May Be Key Inflammation Regulator and Relevant to Poor Prognosis in Adamantinomatous Craniopharyngioma
}

\section{Jia Yibin}

Fourth Military Medical University https://orcid.org/0000-0002-5483-3847

\section{Wang Guanyi}

Fourth Military Medical University: Air Force Medical University

\section{Ye Yuqin}

xi jing yi yuan: Xijing Hospital

\section{Kang Enming}

xi jing yi yuan: Xijing Hospital

\section{Wu JunFeng}

xi jing yi yuan: Xijing Hospital

\section{Wang Jiayou}

xi jing yi yuan: Xijing Hospital

\section{Chen Xiaoyan}

xi jing yi yuan: Xijing Hospital

HeXiaoSheng ( $\nabla$ hexiaos@fmmu.edu.cn )

xi jing yi yuan: Xijing Hospital

\section{Research Article}

Keywords: Craniopharyngioma, surgery, inflammation

Posted Date: October 18th, 2021

DOI: https://doi.org/10.21203/rs.3.rs-977629/v1

License: (c) (1) This work is licensed under a Creative Commons Attribution 4.0 International License. Read Full License 


\section{Abstract}

Craniopharyngioma is one of the most prevalent sellar tumors in children. Though normally, gross resection might be reached, while the prognosis and outcome of the patient is much more worse than any other benign tumor. Inflammation in tumor is of essential in tumor growth and progression. We found that inflammation was relevant to patient outcome and macrophages in adamantinomatous craniopharyngioma were activated in an interesting pattern. We then evaluated immune microenvironment in adamantinomatous craniopharyngioma and intended to screen out potential functional molecules for therapeutic targets and predicting prognosis. The results showed that SERPINs family, especially SERPINE1 and SERPING1 were up-regulated in adamantinomatous craniopharyngioma and might be related to patient outcome in malignant tumor. At the same time, the immune environment of adamantinomatous craniopharyngioma was similar with glioma rather than other benign brain tumors. The study firstly proposes the view that ACP might share the same characteristics with malignant brain tumor, and meanwhile preliminarily demonstrates SERPINs, especially SERPINE1 might also play a critical role in ACP, just like other aggressive cancer.

\section{Introduction}

Craniopharyngioma (CP) is considered as one of most prevalent sellar tumors in children.

Adamantinomatous craniopharyngioma (ACP), one of two CP subtypes, is usually associated with worse clinic outcome and higher rate of recurrence.[1] There used to remain controversy in decision on surgical resection extent, and it seems that more scholars reach the agreement that sub-resection aimed at critical structure protection, followed with post-surgical adjuvant therapy has much better outcome for patients. [2-4] Thus, the dilemma makes it essential to control the progress of tumor effectively whenever pre- or post-surgery.

In recent years, tumor immune microenvironment (TME) and the connection between inflammation and cancer become most highlighted project in the mechanism study.[5] Various cytokines and immune cells are enrolled in this crosstalk. For instance, tumor associated macrophages (TAMs), have been considered as key players in the re-structuring of TME, and its two phenotype, M1 and M2, usually show as anti- or pro-tumor type. Notwithstanding, there are a host of M1 macrophages observed in TME in progressive tumor, implicating much more obscure immune suppressing exists.[6-7] More notably, researchers found the secretion pattern of ACP is much more different from other pediatric benign brain tumor.[8] Similar with kinds of malignant type, ACP is characterized with intense inflammatory response,[8-10] while the key player is still unknown.

It's well known that serine protease inhibitors (SERPINs) family, function mainly as inhibiting serine protease, and a few serpins can also inhibit caspase and some cysteine proteases. Even more, some serpins do not show any protease inhibitory effect but are presented as other functions. SERPINB family has been found to have a strong correlation to especially epithelial tumor, and SERPINE1 (PAl-1) has much more complex function in cancer progression and metastasis.[11-13] In spite of the fact that uPA, 
coupled with PAl-1, have been treated as reliable biomarkers and prognosticators in many cancer types, clear and definite role of the genes are still hiding behind mask.[14]

In this study, we intend to evaluate the relationship of ACP patients' outcome, with inflammation in tumor and resection of surgery, aiming at screening out potential members in tumor immune environment, exploring their function and discussing the feasibility in further clinic application .

\section{Material And Method}

\section{Patients and Tumor Tissue Samples}

This study was approved by the Ethic Committee of Xi Jing Hospital, and it was conducted according to the principles expressed in the Declaration of Helsinki.

The clinical data of $61 \mathrm{CP}$ patients who were operated and pathologically diagnosed in Xi Jing Hospital from 2010 to 2020 were collected. Through outpatient clinic follow-up, telephone follow-up and other ways to acquire the prognosis and survival information of the patient. The age, gender, time of initial or recurrence, surgical approach, operation and anesthesia time, hospital stay, postoperative hormone and electrolyte test indexes, tumor pathological type were also gathered. The extent of tumor resection was evaluated according to the intraoperative observation and the scans of head CT or/and MRI. Two groups, gross total resection (GTR) and subtotal resection (SR), were produced based on the extent of tumor resection. The progress-free survival (PFS) was counted from the day of surgical operation till the day of death, relapse or the last follow-up. 25 ACP patients which were available for paraffin tissue were collected for further inflammatory grading analysis.

\section{Data Source and Processing}

The clinical baseline and mRNA data of patients from TCGA cohort were downloaded from the GEPIA2 (gepia2.cancer-pku.cn).[15] Transcriptional expression profile of ACP, GSE94349, was download from GEO database (ncbi.nlm.nih.gov/geo).[8] GSEA (4.0.3) were used for pathway enrichment and hub genes selecting. TIMER (timer.cistrome.org/) and Oncomine database (oncomine.org) were used to visually display the mRNA levels of certain molecule in Pan-Cancer way.[16-17] CIBERSORT (cibersortx.stanford.edu) and TISCH (tisch.comp-genomics.org) were used to comprehensively visualize and explore the TME.[18-19] Using STRING (string-db.org) to explore the protein-protein interaction (PPI) network.[20] All datasets were retrieved from the published literature, so it was confirmed that all written informed consent was obtained.

\section{Grading of Inflammatory Response In Craniopharyngioma}

Two pathologists evaluated the inflammation grades of ACP in a single-blind way. According to the number of inflammatory cells near the junction of tumor and normal tissue, the evaluation method is executed as follows:[21] (1) Continuously observe 10 fields at the junction between the solid and nonsolid part of tumor tissue under 400 times light microscope and count the inflammatory cells in each 
high-power field of vision; (2)The inflammatory cells were averaged in the above 10 fields, and this average was re-averaged between the two pathological observers. The final score of inflammatory cells was used to make a grading, ie, $\square$ Grade 0 (inflammatory cells is zero), Grade 1 (inflammatory cells $<15$ ), Grade 2 (inflammatory cells $15-50$ ), Grade 3 (inflammatory cells $>50$ ) $\llbracket$. The tissue sample would be reevaluated if its inflammatory cell number reported by the two observers showed a deviation of 10 cells or more. The patients were divided into three subgroups by their inflammatory grade of tumor: (1) Low inflammatory response group with Grade 0-1; (2) Medium inflammatory response group with Grade 2; (3) High inflammatory response group with Grade 3.

\section{HE and IHC/IF Staining}

For HE staining, all sections were processed in the following procedures: (1)Dewaxed in routine, dehydrated in gradient alcohol;(2)Dyed with hematoxylin for 8 mins, washed with running water and 1\% hydrochloric acid alcohol for color separation, slightly washed with water until returning to blue, rinsed with phosphate buffer for 5 min; (3) Eosin staining for 1 min; (4) Dehydrated, transparent and neutral resin sealed; (5)observed under microscope, photographed.

For immunofluorescence staining, all tissue sections underwent the following steps: (1) Dewaxed, dehydrated; (2) Immersed in fresh $0.05 \%$ Triton $\times 100$ for 20 min at $100{ }^{\circ} \mathrm{C}$ with the membrane broken, incubated in $3 \%$ hydrogen peroxide for $30 \mathrm{~min}$; (3)Endogenous peroxidase was removed and washed with PBS for 3 times with each time $5 \mathrm{~min}$; (4) The antigen was repaired by microwave and serum blocking solution was added for $1 \mathrm{~h}$; (5) After incubated at $4{ }^{\circ} \mathrm{C}$ for $24 \mathrm{~h}$, the sections were washed with PBS for 3 times; (6) After incubated for 4 hours at room temperature, they were dyed for 5 mins in DAPI staining solution; (7) After cleaned, sections were sealed with anti-fluorescence quencher medium and photographed. PBS was used as negative control instead of primary antibody.

\section{Paraffin-Fluorescence Probe FISH}

Tissue block was cleanly washed and then immediately placed in the fixed fluid (DEPC) about $12 \mathrm{~h}$ for fixation. The tissue was dehydrated and sections were produced before dewaxed, dehydrated, and digested. Sections were added with pre-hybridization solution and incubated for $1 \mathrm{~h}$ at $37^{\circ} \mathrm{C}$. Next, remove the pre-hybridization solution, then add the probe hybridization solution. Then the sections were incubated in a humidity chamber and hybridized for overnight. Remove the hybridization solution. The sections were washed respectively in $2 \times S S C$ for $10 \mathrm{~min}$ at $37^{\circ} \mathrm{C}$, in $1 \times S S C$ two times for 5 min each at $37^{\circ} \mathrm{C}$, and then in $0.5 \times \mathrm{SSC}$ for $10 \mathrm{~min}$ at room temperature. The following steps are the same to the IF staining procedures.

\section{Statistical Analysis}

The relationship between inflammation grades and clinic characteristics of ACP patients was evaluated by Chi-squared test. The gene expression and cases of complications was compared between 2 groups by Student $t$ test. Multiple comparisons of immune cells infraction were performed by Tukey's multiple 
comparisons test, followed by One-Way ANOVA. Kaplan-Meier curves were constructed to compare progress-free survival (PFS), overall survival (OS) and disease-free survival (RFS) in patient subgroups, and the significance was determined by log-rank test. In CIBERSORT and GSEA, permutations for significance analysis were set as 100 times. Correlations were assessed by Person correlation analysis. Clinical data statistical analyses were carried out using SPSS Statistics 24.0. All statistical tests were 2tailed and $P<0.05$ was considered statistically significant

\section{Results}

\section{Extent of Resection Seems to Show no Difference in Post-Operation Complications and Prognosis, While Inflammatory Response in ACP is Relevant to Poor Clinical Outcome}

In 61 cases, total resection was performed in 36 cases and subtotal resection in 25 cases (residual part adhered to important structures, tumor or cystic part that is hard to separate) (Talbe.1). By comparing the differences of different resection methods and different surgical approaches, it was observed that there was no significant difference between postoperative complications and long-term no progress of children (Table. S1 and S2), in spite of relapse tissue shows more papillary humps embedded in tissue (Figure S1).

At the end of the follow-up, there were tumor recurrences in 18 patients among all the 59 survived, and the rest two died of electrocytes imbalance and hypothalamus reactions during admission. The relapse time was 6-70 (median 24) months, and the follow-up time was 1-120 (median 21) months. There was no significant difference in recurrence rate in the gender $(P=0.515)$, and in surgical approaches $(P=0.381)$. PFS calculated by Kaplan Meier analysis and log rank test revealed that there was no significant difference between the degree of tumor resection and PFS of patients with progression free survival ( $P=$ $0.693)$, that is, compared with the total resection group, the recurrence rate of patients with partial tumor resection was not increased (Figure. 1A).

Among the 25 patients who were diagnosed as ACP by postoperative pathology, there were 3 cases of low-grade inflammatory response, 12 cases of medium-grade and 10 cases of high-grade, $\chi 2$ test showed that there was no significant difference in the degree of surgical resection between different inflammatory grades $(P=0.276)$, but there was a high correlation between the degree of surgical resection and tumor progression $(P<0.001)$. Meanwhile, the survival analysis showed that the prognosis of high-grade group was significantly worse than that of low-grade group ( $P=0.022)$ (Figure. 1B). At the same time, in 6 cases of recurrent tumor tissue, 5 cases were high inflammatory infiltration state. Comparison before and after recurrence of the same case, showed more M2 activated phenotype of macrophage and microglia, while it's interesting to find that Iba- $1^{+}$cells seem to decrease in relapse tumor (Figure. 1C and 1D). All these results suggested inflammatory response might influence the progress of ACP.

\section{Macrophage and Serpins Family are Potential Players in TME of ACP}


The results above hinted that inflammation might influence the progress of ACP and patients' outcome. On the evidence that macrophage and microglia in brain are critical in TME re-building. In GSE94349, we used NetworkAnalyst and GSEA to screen potential functional molecules associated with TAMs and inflammation (Figure 2A; Figure S1B), and it indicated that SERPINs family (SERPINB2, SERPINB5, SERPINE1, SERPING1) were respectively involved in various functional gene sets like macrophage, microglia, inflammatory response and classic inflammation pathway NF-KB (Figure 2B). Apart from the four genes, we chose top 5 genes in each gene set to demonstrate their relationship. Correlation hotmap showed the potential relationship among those hub genes in different gene sets, and SERPINE1 had significant correlation with SERPINB2, SERPINB5. What's more, SERPINE1 was also associated with either PRRG4, TNFSF10, DSC2 or SDC1, suggesting a close relationship of these five genes with tumor proliferation, invasion and metastasis (Figure 2D).

And it was worth noting that expression level of SERPING1 seemed to show negative correlation with other genes, suggesting it might participate in different pathways from SERPINE1. IL15, known as functional immune inducing and activation molecules, also showed pertinent connection with SERPINE1, SERPINB2 and SERPING1. We next used CIBERSORTx to verify the immune environment of ACP, PA and normal brain tissues, and the results showed high correlation and percentage of M0 macrophage, M2 macrophage, $\mathrm{CD} 4^{+} \mathrm{T}$ memory cells, which accounted for more than $60 \%$, indicating involvement of macrophage in tumor environment (Figure 2C). Multiple comparisons between immune cells infraction of $\operatorname{ACP}(n=24), P A(n=10)$ and normal brain $(n=27)$ tissues displayed macrophages M0, T cells CD $4^{+}$ memory resting and plasma cells in ACP were significantly enriched. Macrophages M2 in tumor were all up-regulated, while macrophages $\mathrm{M} 1$ and $\mathrm{T}$ cells $\mathrm{CD} 4^{+}$memory activated were both less activated in $\mathrm{ACP}$ and PA (Figure 2E).

\section{Comprehensive Analysis of SERPINs Family in Glioma and HNSC Shows SERPINs Prognostic Values}

Based on the evidence that TME similarity with brain invasive tumor,[10] and ACP are tendentious to malignantly transform into squamous cancer, we compared the transcriptional levels of SERPINs family in tumors with normal tissue in Oncomine database ( $P$ Value $<0.01$, Fold Change $>1.5$, Top Gene $10 \%$, Data Type mRNA) (Figure 3B). It showed SERPINE1 was significantly upregulated in Brain, HNSC and Esophageal Cancer. SERPINB2 and SERPINB5 were more tendinous increasing in Lung Cancer and Gastrointestinal Cancer. The expression level of SERPING1 was higher in Brain, Esophageal Cancer and Lymphoma. The mRNA expression levels of SERPINE1 were significantly in patients of glioma and squamous cancer (Table S3).

In addition, Pan-Cancer analysis in TIMER also showed that the family increase in various cancer like COAD, HNSC, LUSC $(P<0.001)$ (Figure 3A). We also tried to find if there exist similar expression patterns of SERPINs in other relevant cancer type. In GEPIA we chose GBM, LGG and HNSC to further explore transcriptional level of SERPINs between tumor and normal tissue ( $\left|\log _{2} \mathrm{FC}\right|$ Cutoff 1.5, q-value Cutoff 0.01). it was found that SERPINE1 and SERPING1 were significantly upregulated in all three cancers. In HNSC SERPINB5 was higher in tumor tissues while SERPINB2 was lower than normal tissue (Figure 3C). 
Correlation between SERPINs expression level and tumor grades (stage) revealed that SERPINs had significant relevance with grades of GBM, while it seemed to have no influence in HNSC (Figure 4A). Kaplan-Meier curves demonstrated SERPINE1, SERPINB2 and SERPING1 were associated with RFS but not OS in GBM patients $(P<0.05)$ (Figure 4B). And all SERPINs had no statistical difference both in OS and RFS in HNSC patients (Figure S3).

\section{Predicted Functions and Pathways of the Changes among SERPINs and SERPINs in Macrophages Might Shares Similar Expression Pattern between ACP and GBM}

Using TISCH, we sourced single-cell datasets GSE141982 and GSE131928 to reach a comprehensive exploration of SERPINs and TME. Cell cluster profiles showed that SERPINE1 evidently collocate in macrophage and mononuclear clusters, with slight enrichment in malignant cells and less in other cell types (Figure 5A). At the same time, SERPING1 had a minor expression level. SERPINB2 and SERPINB5 were both expressed with basic levels in all kinds of cells (Figure 5B). Gene-gene correlation showed SERPINE1 had a certain relation with SERPINB2 and SERPING1. Besides other SERPINs, SERPINE1 also showed significant correlation with CD68, CD86, CD163 and CD206 (Figure 5C). PPI network among SERPINs had been established to explore the relationship between upstream and downstream connection and probable pathway involved with tumor metastasis, invasion and activation of macrophage (Figure $\mathrm{S} 2 \mathrm{C}$ ). Enrichment pathway consist of various tumor inflammation pathway, such as p53, angiogenesis and so on. At last, we tried to conduct FISH to validate whether there exists colocation between SERPINEs and macrophage in ACP, and the result showed there existed evident accumulation of SERPINE1 in macrophage. It was notable that SERPINE1 $1^{+}$macrophages were more likely located surrounding solid tumor while SERPING $1^{+}$in cystic part. (Figure 5D).

\section{Discussion}

ACP is one of prevalent sellar tumor, especially among children, which usually results in severe visual impairment, dysplasia and endocrine disorders. Worse primary location and poor prognosis, require that more comprehensive knowledge and systemic treatment are needed in ACP patients.[1] We provide a supplementary confirmation on the viewpoint that sub-resection may not harm patients' prognosis, compared with total-resection. At the same time, reducing the extra damage caused by surgical intervention might be helpful to improve the endocrine condition of patient. And accompanied with adjuvant therapy, ideal therapeutic target can be accomplished. It's also been hypothesized that inflammation in ACP may influence the progress of the tumor, while mechanism behind is still unknown. Current understanding is normally about severe tissue adhesion and calcification, which indirectly affected the difficulty of surgery.[22] IF results show more M2 macrophages are activated and recruited in recurrent tumor, compared with the primary, while Iba- $1^{+}$cells were less in recurrent tumor. We conjecture new TME provides a more appropriate condition for ACP growth by immunity inhibiting, strengthening the evidence that inflammation might influence the progress of ACP. 
Similar fierce inflammation response with malignant TME hints that ACP may share a certain series of molecules associated with tumor progress or recurrence.[8-9, 21] Uncovering the behind mechanism and intending to screen definite molecules are critical. The role of inflammation in tumor has been debated for a long time, and in paradigm, researchers usually categorize inflammation into pro- or anti- types, so as TAMs,[6] which are recognized as M1 or M2 phenotype. It's intelligible that M2 are more predominant in tumor with progressiveness. However, as matter of fact, M1 type is also abundant in various tumor tissue, and probably associated with tumor metastasis.[23] In GSE94349, we use CIBERSORT to evaluate immune cells infraction in ACP, normal brain tissues and PA, the most prevalent pediatric brain tumor. Results show percentage of MO macrophages in ACP are much higher than PA and normal brain. This kind of immune inhibiting mechanism needs further exploration and more comprehensive understanding beyond traditional classification based on surface markers is needed. We also apply the same series for difference expression genes and gene set enrichment analysis. It shows various immune and inflammatory pathway are activated in ACP. Among those hub genes, we have noticed that SERPINs are significantly enriched. TME analysis shows macrophages are prominent in ACP tissue and SERPINs family (SERPINE1, SERPINB2, SERPINB5 and SERPING1), which are closely related to various inflammatory mediators, might participate into the reaction in some way.

Apart from the evidence that ACP shows more aggressive biological behavior than any other benign brain tumors and characterize as malignant clinic course,[10] there also exists kinds of viewpoint that the tumor might result in gliosis,[24-25] or the reactive ependymoma appearing after partial resection of a craniopharyngioma and repeated postoperative radioherapy.[26-27] What's more, SERPINE1 has already been confirmed as regulator of glioblastoma growth and invasion.[28] Therefore, it is speculated that ACP may share kinds of certain biological process similar with malignant tumor like glioma. And we attempt to validate clinic value of SERPINs for expression level and prognosis prediction in glioblastoma. In the present study, it's found that all four SERPINs are overexpressed in GBM and significantly associated with cancer progress. Single cells profiles show SERPINE1 is evidently enriched in macrophages, providing the proof about its intervention in recruitment and polarization of TAMs. Function verification experiments are needed in further research.

SERPINE1 (plasminogen activator inhibitor-1, PAl-1) is upregulated in kinds of tumors, and has been recognized as a strong predictor of poor outcome and worse response to treatment.[11, 14] Apart from inducing angiogenesis, it also has been found be able to promote the recruitment of macrophage and M2 polarization. SERPINE1 is also observed to be effective in the migration of mononuclear cells, thus it's probably that in ACP the SERPINE1 function as key driver to recruit macrophage and other immune cells to tumor, which then proceed the re-building of TME. And the complex crosstalk between PAI-1, IL-6, IL-10 and other cytokines make it need more precise experiment to clarify certain mechanism.[11, 29] In this study, we found SERPINE1 has potential prediction value and may has similar expression profile in ACP and GBM, suggesting the molecule can be treated as target in prognosis prediction or therapy.

SERPINB2 and SERPINB5 have been recognized as tumor suppressor genes, especially in gastrointestinal and other epithelial tumor.[12-13, 30] Theoretically, downregulation of these genes may lead to tumor 
growth invasion and metastasis, and some studies indeed have verified it. However, similar with SERPINE1, SERPINB2 and SERPINB5 seem not simply downregulated in tumors, both two can also have higher transcriptional levels in some epithelial-origins tumors. This paradoxical phenomenon reveals the complexity of TME, and further exploration is needed.[11] What's more, SERPING1, normally having negative correlation with clinic outcome in other cancer or immune dysregulating disease,[31-32] shows positive correlation with other genes in GBM while negative in ACP. Thus, we hypothesize SERPING1 has a more intricate crosstalk in inflammation mediator network.

In summary, the article provides further evidence for inflammation in ACP, revealing the possibility of potential functional molecules for treatment target and prediction of prognosis. Treatment targeting SERPINs is still challenging, for definite roles of them are still sophisticated, while it's reasonable to use them as supplementary predication markers. The study firstly proposes the view that ACP might share the same characteristics with malignant brain tumor, and meanwhile preliminarily demonstrates SERPINs, especially SERPINE1 might also play a critical role in ACP, just like other aggressive cancer. Further validating experiments are needed, in spite of difficulties that lack of stable ACP cell lines in vitro and animal models. We hope our data can provide additional clues and evidence for research in ACP inflammation and at last, enhancing the welfare of ACP patients.

\section{Declarations}

\section{Ethics approval and consent to participate}

The collection of the patient clinic data and samples analysis was approved by the ethics committee of Xijing Hospital (KY20212135-F-1). Our study adhere to the tenets of the 1964 Declaration of Helsinki. Informed consent was obtained form all the patients.

\section{Consent for publication}

Not applicable.

\section{Competing Interest}

The authors declare no competing interests.

\section{Funding}

The present work was supported by the Natural Science Foundation of China (NSFC) grant to Xiaosheng $\mathrm{He}$ (Nos. 81971156 and 2020XC015)

\section{Author Contributions}

Y. J. and G. W. designed the study. J. W.and E. K. collected the data, carried out data analyses. Y. Y. produced the initial draft of the manuscript. Y. J. and G. W. contributed to drafting and finishing the 
manuscript. J. W. and X. C. were responsible for pathologic and histologic experiments. All authors had read and approved the final submitted manuscript.

\section{Acknowledgements}

We thank Dr. Donson for the generosity of data sharing.

\section{Availability of data and material}

The datasets used or analysed during the current study are available from the corresponding author on reasonable request.

\section{References}

1. Muller HL, Merchant TE, Warmuth-Metz M, Martinez-Barbera JP, Puget S. Craniopharyngioma. Nat Rev Dis Primers 2019; 5: 75. DOI: 10.1038/s41572-019-0125-9.

2. Muller HL. Consequences of craniopharyngioma surgery in children. J Clin Endocrinol Metab 2011; 96: 1981-1991. DOI: 10.1210/jc.2011-0174.

3. Muller HL. More or less? Treatment strategies in childhood craniopharyngioma. Childs Nerv Syst 2006; 22: 156-157. DOI: 10.1007/s00381-005-1192-7.

4. Sadashivam S, Menon G, Abraham M, Nair SN. Adult craniopharyngioma: The role of extent of resection in tumor recurrence and long-term functional outcome. Clin Neurol Neurosurg 2020; 192: 105711. DOI: 10.1016/j.clineuro.2020.105711.

5. Hui L, Chen Y. Tumor microenvironment: Sanctuary of the devil. Cancer Lett. 2015; 368: 7-13. DOI: 10.1016/j.canlet.2015.07.039.

6. Pathria P, Louis TL, Varner JA. Targeting Tumor-Associated Macrophages in Cancer. Trends Immunol. 2019; 40: 310-327. DOI: 10.1016/j.it.2019.02.003.

7. Hambardzumyan D, Gutmann $\mathrm{DH}$, Kettenmann $\mathrm{H}$. The role of microglia and macrophages in glioma maintenance and progression. Nat. Neurosci. 2016; 19: 20-27. DOI: 10.1038/nn.4185.

8. Donson AM, Apps J, Griesinger AM, Amani V, Witt DA, Anderson R, et al. Molecular Analyses Reveal Inflammatory Mediators in the Solid Component and Cyst Fluid of Human Adamantinomatous Craniopharyngioma. J Neuropathol Exp Neurol 2017; 76: 779-788. DOI: 10.1093/jnen/nlx061.

9. Whelan R, Prince E, Gilani A, Hankinson T. The Inflammatory Milieu of Adamantinomatous Craniopharyngioma and Its Implications for Treatment. J. Clin Med 2020; 9. DOI: 10.3390/jcm9020519.

10. Petralia F, Tignor N, Reva B, Koptyra M, Chowdhury S, Rykunov D, et al. Integrated Proteogenomic Characterization across Major Histological Types of Pediatric Brain Cancer. Cell 2020; 183: 19621985. DOI: 10.1016/j.cell.2020.10.044.

11. Kubala MH, DeClerck YA. The plasminogen activator inhibitor-1 paradox in cancer: a mechanistic understanding. Cancer Metastasis Rev 2019; 38: 483-492. DOI: 10.1007/s10555-019-09806-4. 
12. Bodenstine TM, Seftor RE, Khalkhali-Ellis Z, Seftor EA, Pemberton PA, Hendrix MJ. Maspin: molecular mechanisms and therapeutic implications. Cancer Metastasis Rev 2012; 31: 529-551. DOI: 10.1007/s10555-012-9361-0.

13. Dzinic SH, Bernardo MM, Li X, Fernandez-Valdivia R, Ho YS, Mi QS, et al. An Essential Role of Maspin in Embryogenesis and Tumor Suppression. Cancer Res. 2017; 77: 886-896. DOI: 10.1158/00085472.CAN-16-2219.

14. Kubala MH, Punj V, Placencio-Hickok VR, Fang H, Fernandez GE, Sposto R, et al. Plasminogen Activator Inhibitor-1 Promotes the Recruitment and Polarization of Macrophages in Cancer. Cell Rep 2018; 25: 2177-2191. DOI: 10.1016/j.celrep.2018.10.082.

15. Tang Z, Li C, Kang B, Gao G, Li C, Zhang Z. GEPIA: a web server for cancer and normal gene expression profiling and interactive analyses. Nucleic Acids Res. 2017; 45: W98-W102. DOI: 10.1093/nar/gkx247.

16. Li B, Severson E, Pignon JC, Zhao H, Li T, Novak J, et al. Comprehensive analyses of tumor immunity: implications for cancer immunotherapy. Genome Biol. 2016; 17: 174. DOI: 10.1186/s13059-0161028-7.

17. Rhodes DR, Yu J, Shanker K, Deshpande N, Varambally R, Ghosh D, et al. ONCOMINE: a cancer microarray database and integrated data-mining platform. Neoplasia 2004; 6: 1-6. DOI: 10.1016/s1476-5586(04)80047-2.

18. Sun D, Wang J, Han Y, Dong X, Ge J, Zheng R, et al. TISCH: a comprehensive web resource enabling interactive single-cell transcriptome visualization of tumor microenvironment. Nucleic Acids Res. 2021; 49: D1420-D1430. DOI: 10.1093/nar/gkaa1020.

19. Newman AM, Steen CB, Liu CL, Gentles AJ, Chaudhuri AA, Scherer F, et al. Determining cell type abundance and expression from bulk tissues with digital cytometry. Nat. Biotechnol. 2019; 37: 773782. DOI: 10.1038/s41587-019-0114-2.

20. Szklarczyk D, Franceschini A, Wyder S, Forslund K, Heller D, Huerta-Cepas J, et al. STRING v10: protein-protein interaction networks, integrated over the tree of life. Nucleic Acids Res. 2015; 43: D447-D452. DOI: 10.1093/nar/gku1003.

21. Peng J, Yang L, Pan J, Wang C, Nie J, Liu Y, et al. Clinical features and prognosis of pediatric infradiaphragmatic craniopharyngioma relative to the tumor inflammatory response. Pediatr. Res. 2021; 89: 1119-1125. DOI: 10.1038/s41390-020-1013-4.

22. Chen M, Zhang Z, Yang M, Li ST. Prediction of calcification tendency in pediatric cystic adamantinomatous craniopharyngioma by using inflammatory markers, hormone markers, and radiological appearances. Childs Nerv Syst 2019; 35: 1173-1180. DOI: 10.1007/s00381-019-04178-0.

23. Cho U, Kim B, Kim S, Han Y, Song YS. Pro-inflammatory M1 macrophage enhances metastatic potential of ovarian cancer cells through NF-kappaB activation. Mol Carcinog 2018; 57: 235-242. DOI: $10.1002 / m c .22750$.

24. Pettorini BL, Inzitari R, Massimi L, Tamburrini G, Caldarelli M, Fanali C, et al. The role of inflammation in the genesis of the cystic component of craniopharyngiomas. Childs Nerv Syst 2010; 26: 1779- 
1784. DOI: $10.1007 / \mathrm{s} 00381-010-1245-4$.

25. Tena-Suck ML, Morales-Del AA, Hernandez-Campos ME, Fernandez-Valverde F, Ortiz-Plata A, Hernandez $A D$, et al. Ultrastructural characterization of craniopharyngioma at the tumor boundary: $A$ structural comparison with an experimental toxic model using "oil machinery" fluid, with emphasis on Rosenthal fibers. Acta Histochem. 2015; 117: 696-704. DOI: 10.1016/j.acthis.2015.09.006.

26. Tolnay M, Kaim A, Probst A, Ulrich J. Subependymoma of the third ventricle after partial resection of a craniopharyngioma and repeated postoperative irradiation. Clin. Neuropathol. 1996; 15: 63-66.

27. Ho KC, Meyer G, Caya J, Tieu TM, Prentiss A. Craniopharyngioma and "reactive" subependymoma of the third ventricle-a case report. Clin. Neuropathol. 1987; 6: 12-15.

28. Seker F, Cingoz A, Sur-Erdem 0, Erguder N, Erkent A, Uyulur F, et al. Identification of SERPINE1 as a Regulator of Glioblastoma Cell Dispersal with Transcriptome Profiling. Cancers 2019; 11: 1651. DOI: 10.3390/cancers 11111651.

29. Zhou J, Zhang C, Pan J, Chen L, Qi ST. Interleukin6 induces an epithelialmesenchymal transition phenotype in human adamantinomatous craniopharyngioma cells and promotes tumor cell migration. Mol Med Rep 2017; 15: 4123-4131. DOI: 10.3892/mmr.2017.6538.

30. Westrick RJ, Rojkjaer LP, Yang AY, Roh MH, Siebert AE, Ginsburg D. Deficiency of plasminogen activator inhibitor-2 results in accelerated tumor growth. J. Thromb. Haemost. 2020; 18: 2968-2975. DOI: $10.1111 /$ jth. 15054 .

31. Mete GN, Gulbahar O, Onay H, Peker KZ, Ozgul S, Kose T, et al. Deletions in SERPING1 Lead to Lower C1 Inhibitor Function: Lower C1 Inhibitor Function Can Predict Disease Severity. Int Arch Allergy Immunol 2019; 178: 50-59. DOI: 10.1159/000492583.

32. Jovcevska I. Genetic secrets of long-term glioblastoma survivors. Bosn J Basic Med Sci 2019; 19: 116-124. DOI: 10.17305/bjbms.2018.3717.

\section{Table}

Table 1. Baseline information of patients 


\begin{tabular}{|c|c|}
\hline Patient & Cases】\%区 \\
\hline Total & 61 \\
\hline \multicolumn{2}{|l|}{ Gender } \\
\hline Male & $29 \llbracket 47.5 \rrbracket$ \\
\hline Female & $32 \rrbracket 52.5 \rrbracket$ \\
\hline \multicolumn{2}{|l|}{ Age } \\
\hline$\leq 6$ & $24 \llbracket 39.3 \rrbracket$ \\
\hline $6 \sim 12$ & $23 \llbracket 37.7 \rrbracket$ \\
\hline$\geq 12$ & $14 \llbracket 23 \rrbracket$ \\
\hline \multicolumn{2}{|c|}{ Counts Of Lesion } \\
\hline Single & $58 \varangle 95.1 \rrbracket$ \\
\hline Multi & $3 \otimes 4.9 \rrbracket$ \\
\hline \multicolumn{2}{|c|}{ Property Of Lesion } \\
\hline Cystic-Solid & $32 \rrbracket 52.5 \rrbracket$ \\
\hline Cystic & $23 \llbracket 37.7 \rrbracket$ \\
\hline Solid & $6 \otimes 9.8 \rrbracket$ \\
\hline \multicolumn{2}{|l|}{ Surgery Type } \\
\hline Craniotomy & $56 \otimes 91.8 \rrbracket$ \\
\hline Endoscopy & $2 \bigotimes 8.2 \rrbracket$ \\
\hline \multicolumn{2}{|c|}{ Extent Of Resection } \\
\hline Total-Resection & $36 \rrbracket 59 \rrbracket$ \\
\hline Sub-Resection & $25 \llbracket 41 \rrbracket$ \\
\hline
\end{tabular}

\section{Figures}

\section{Figure 1}

Extent of resection seems to show no difference in post-operation complications and prognosis, while inflammatory response in ACP is relevant to poor clinical outcome. (A) K-M curves show total survival data and PFS between sub-resection group and total-resection group. (B) PFS among groups with 
different inflammation grades. (C)(D) IF results show markers of activating macrophages in primary and recurrent ACP tissue. *** $\mathrm{P}<0.001$

A

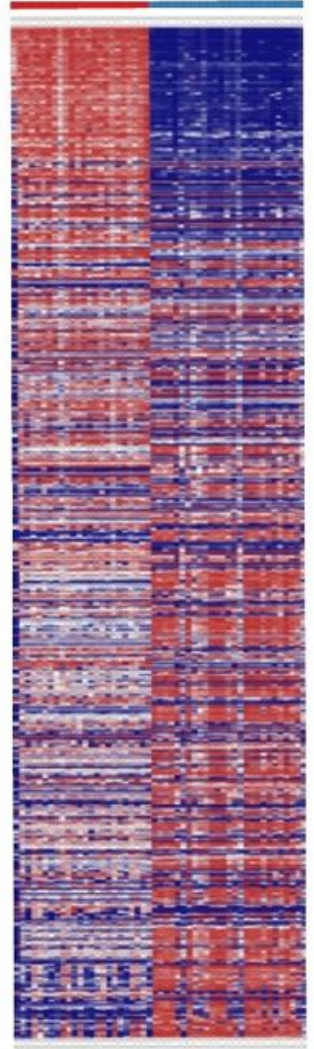

B

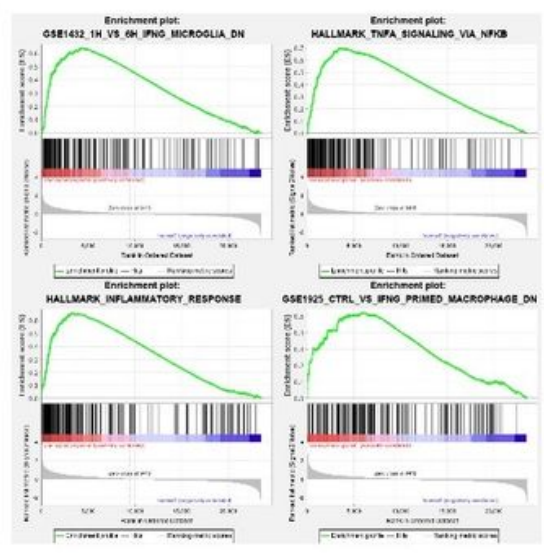

D

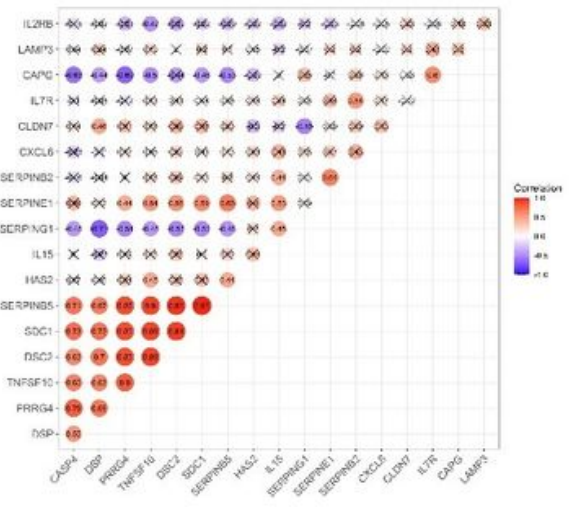

C
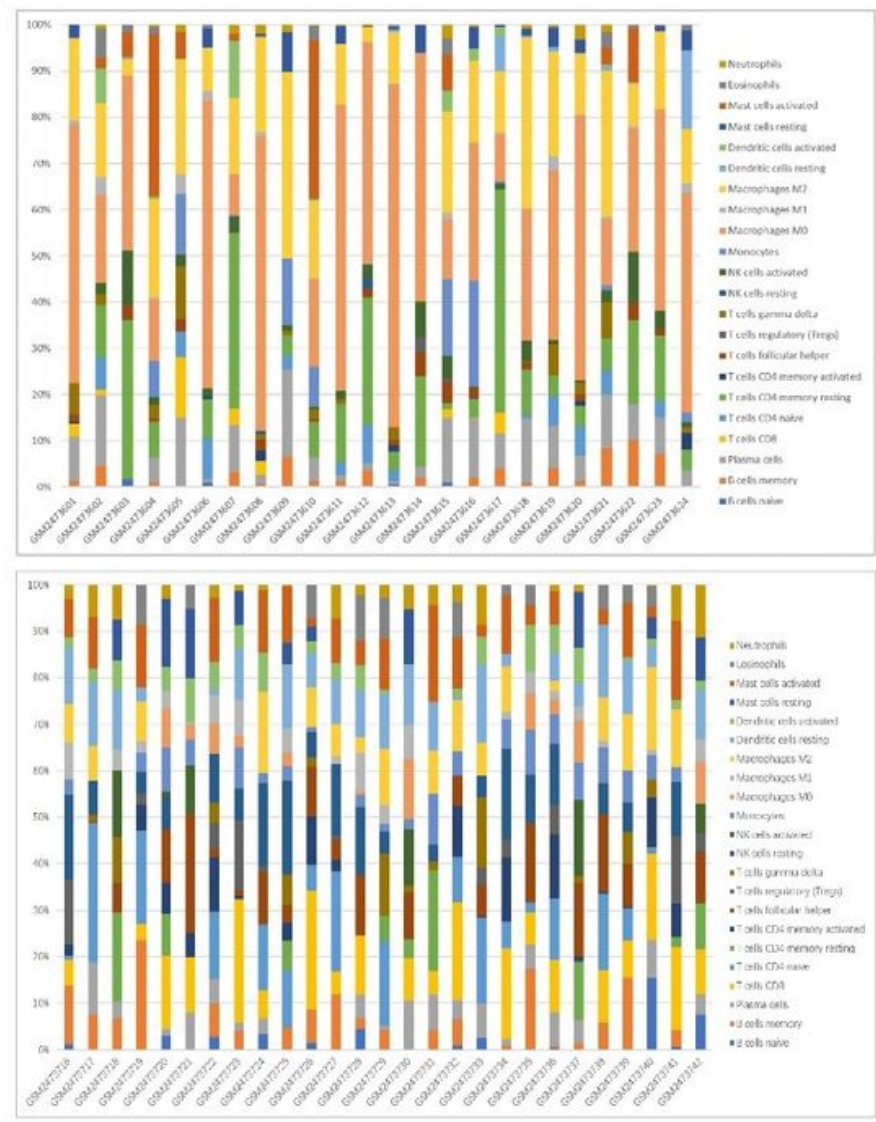

$\mathbf{E}$
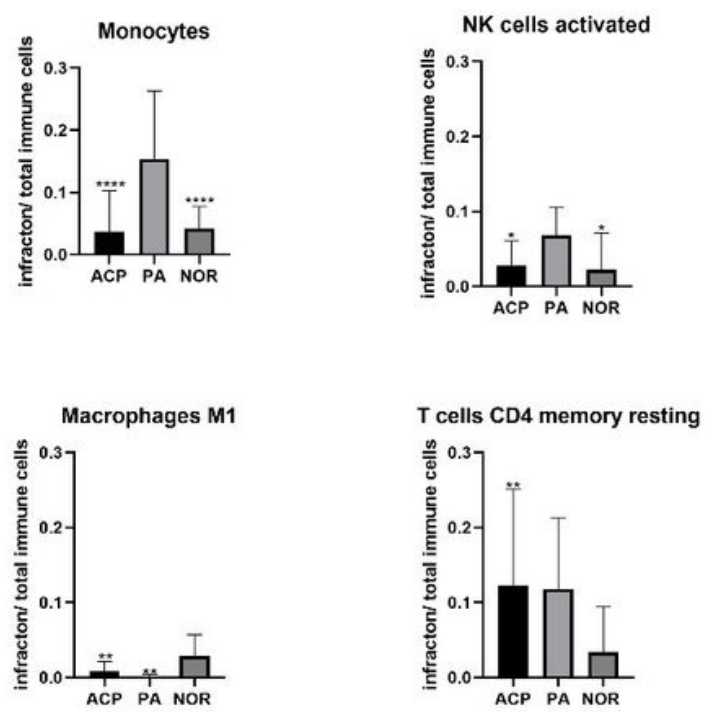
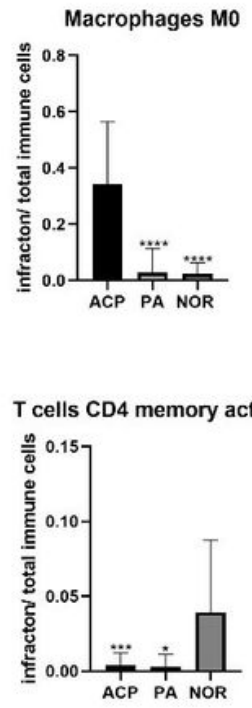
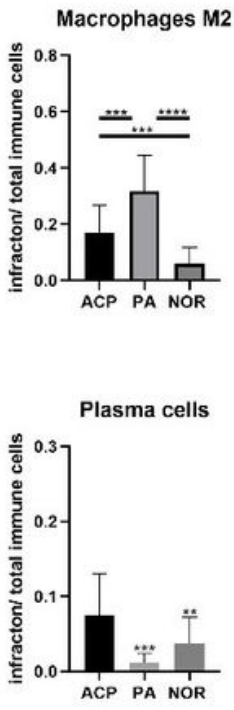

\section{Figure 2}

Macrophage and serpins family are potential players in TME of ACP (A) DEGs between ACP and normal brain tissue. (B) GSEA analysis result show DEGs enrichment in various inflammation-associated pathway. (C) CIBERSORT show high infraction of CD4+ T cells, M0 and M2 macrophages in ACP TME, while the fraction of various immune cells in normal brain tissue shows no significant difference 
(CIBERSORTX). (D) Correlation heat map display gene correlation matrix among top 5 hub genes in each functional gene sets. (E) Multiple comparisons between immune cells infraction of ACP $(n=24), P A(n=10)$ and normal brain $(n=27)$ tissues. ${ }^{*} P<0.05, * * P<0.01, * * * P<0.001, * * * * P<0.0001$

A
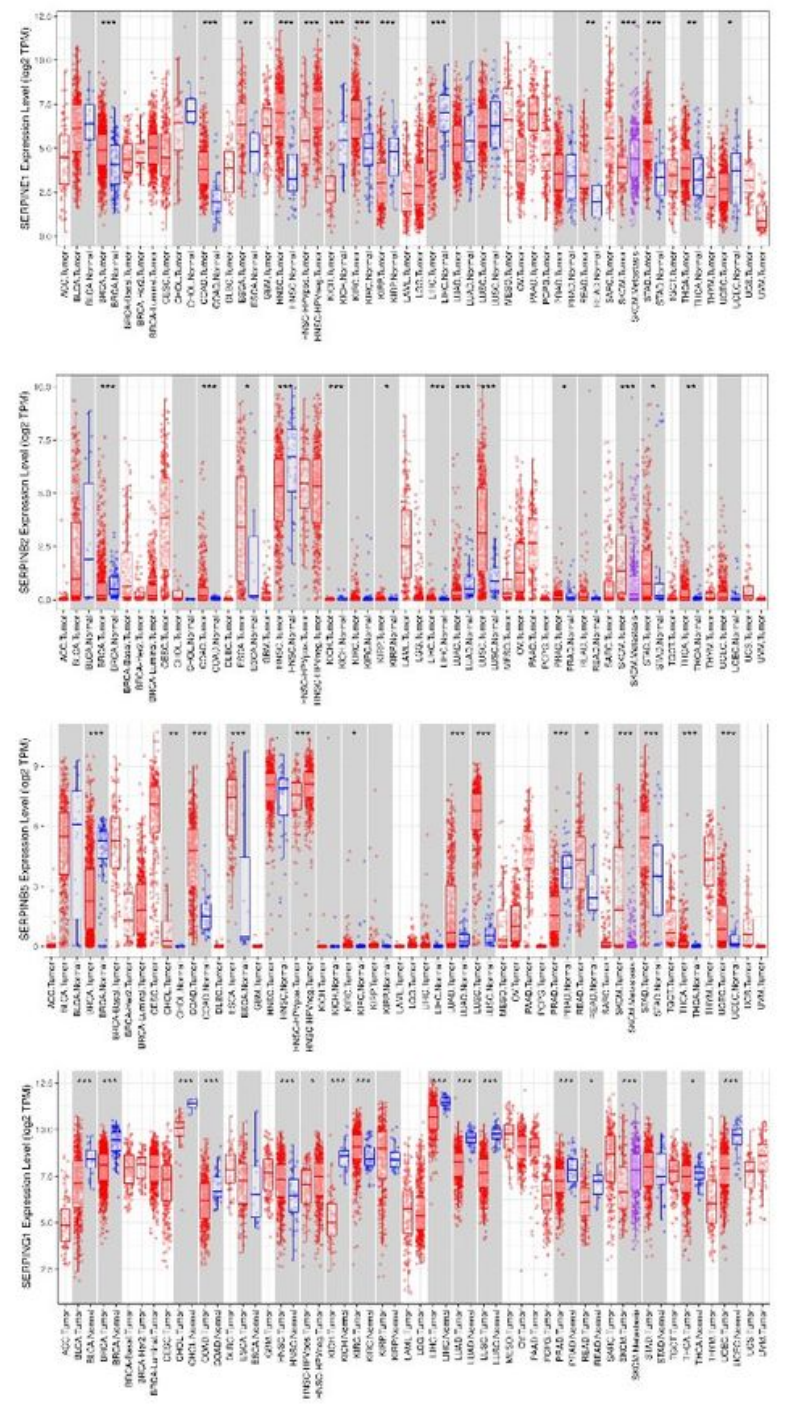

B

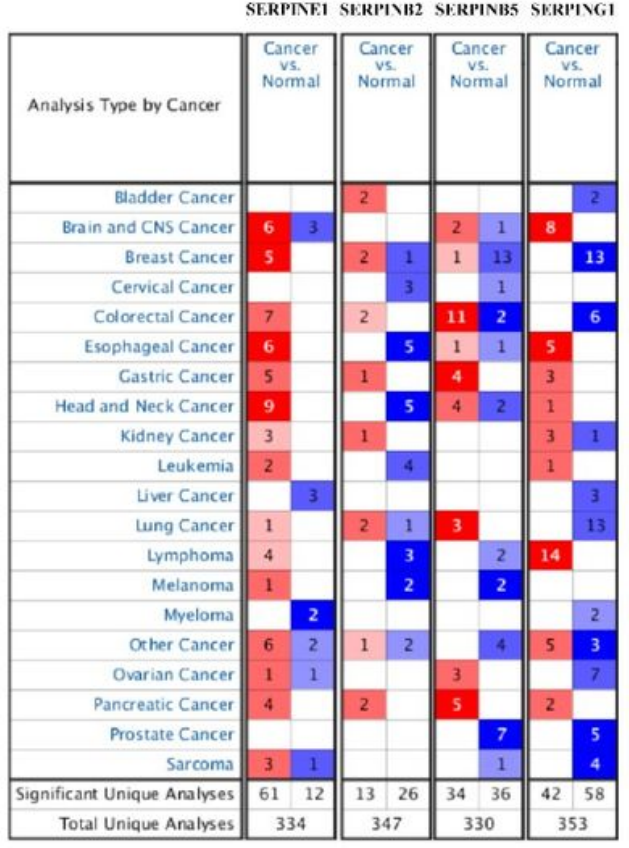

C

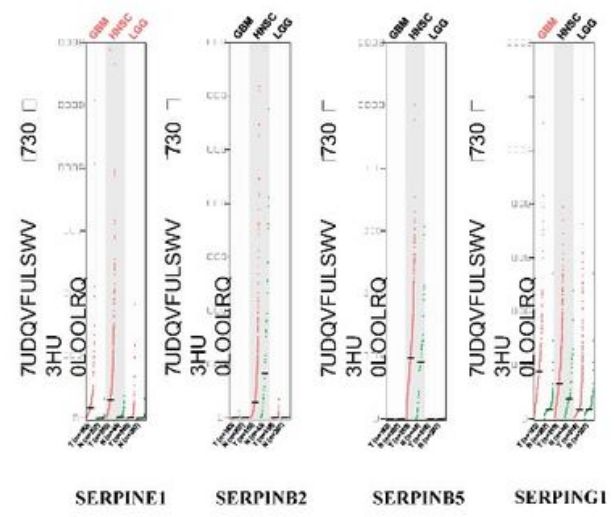

\section{Figure 3}

Comprehensive analysis of Serpins family. (A) Pan-Cancer analysis show SERPINs are upregulated in various cancer (TIMER). (B) The transcription levels of SERPINs in different types of cancers (Oncomine). (C) The expression of SERPINs in GBM, LGG and HNSC (GEPIA). 
A
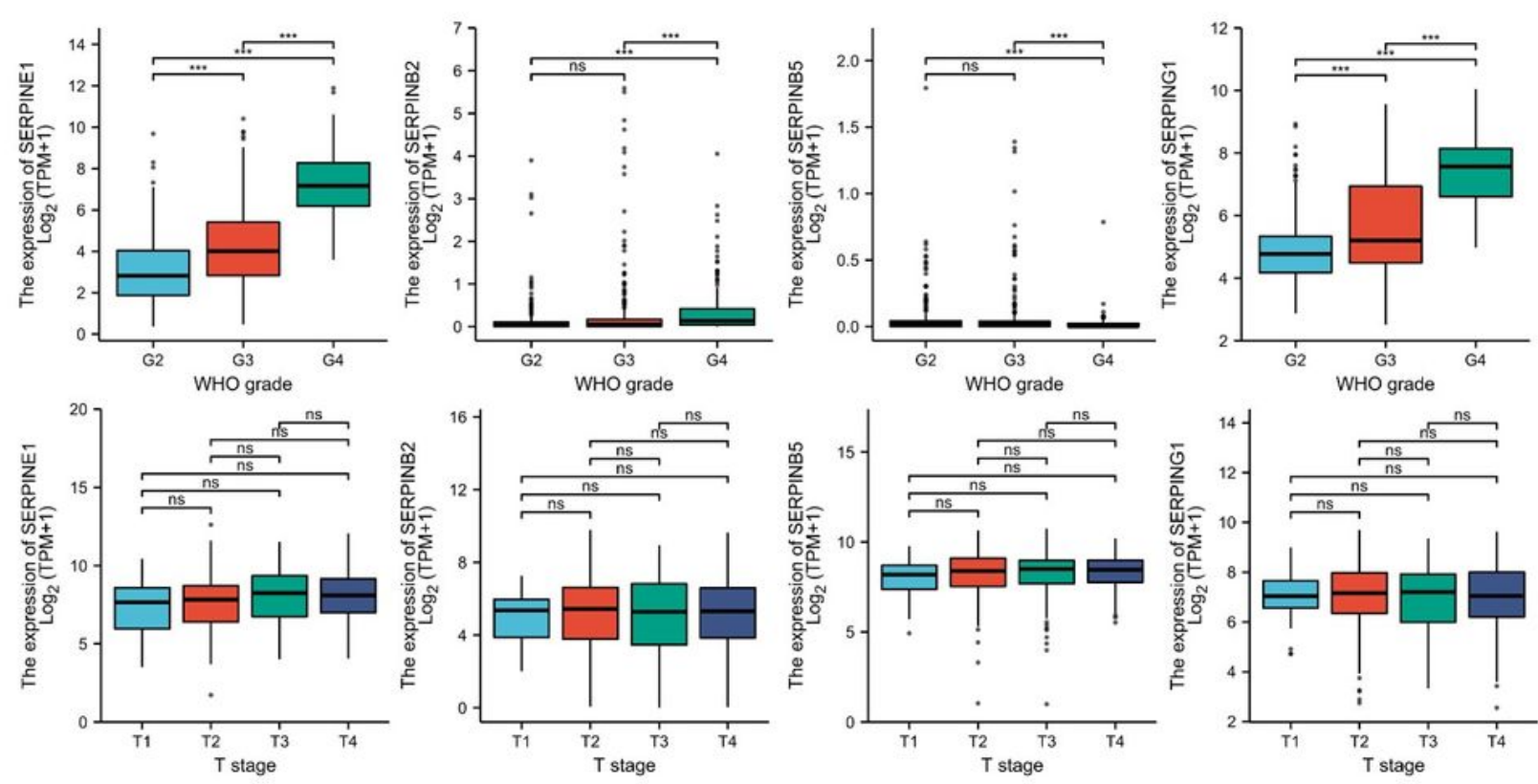

SERPINE1

SERPINB2

SERPINB5

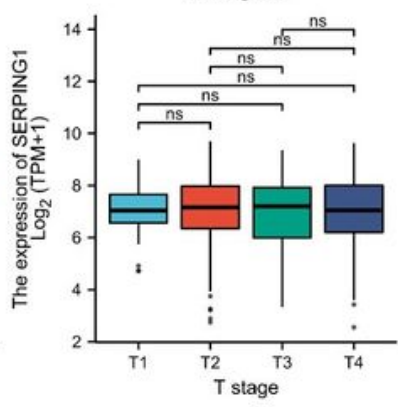

SERPING1

B
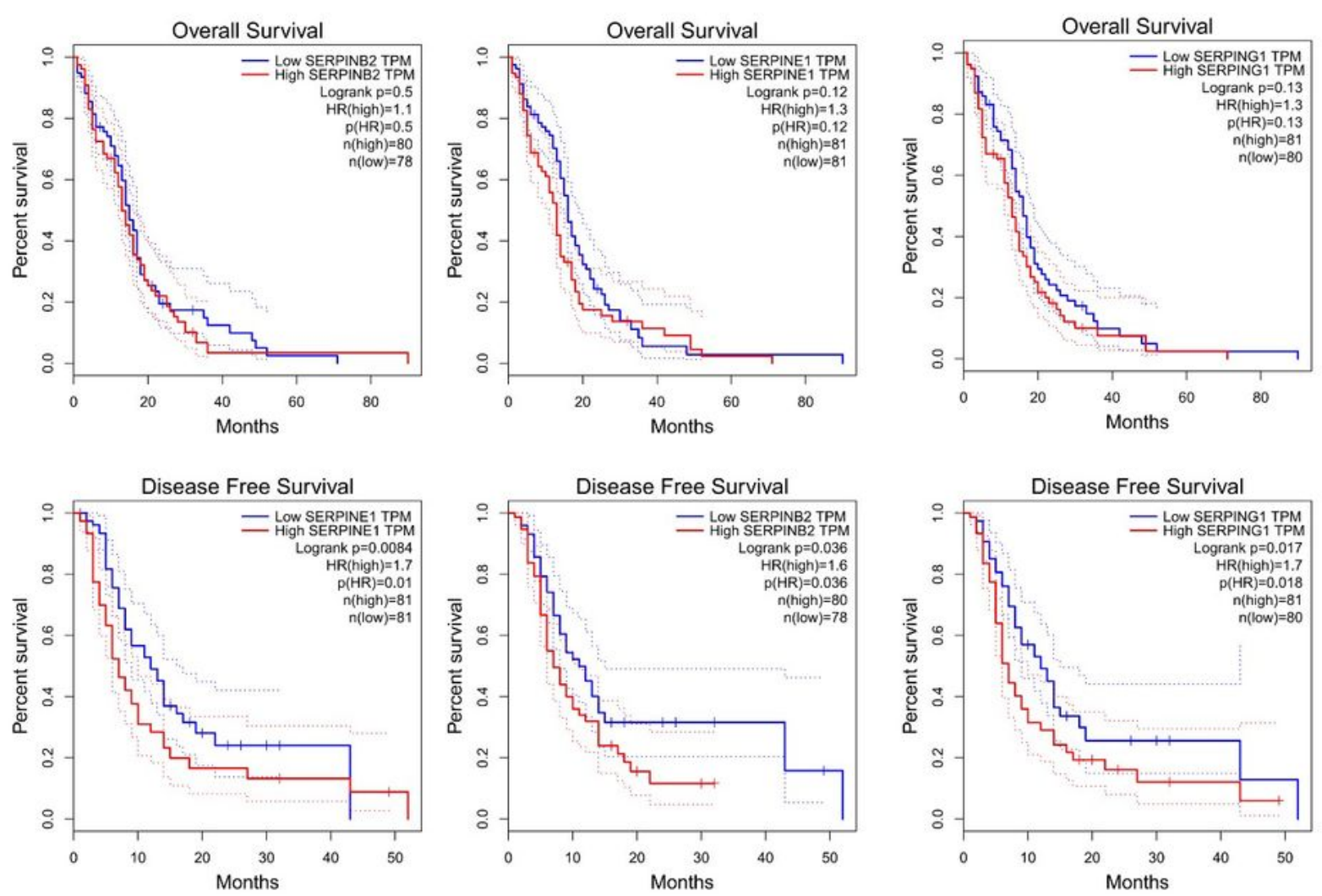

\section{Figure 4}

Prognostic value of SERPINs in GBM and HNSC. (A) Correlation between SERPINS expression and tumor grade or stage in GBM and HNSC patients (GEPIA). (B) The prognostic value of mRNA level of SERPINs in GBM patients (GEPIA) 
A

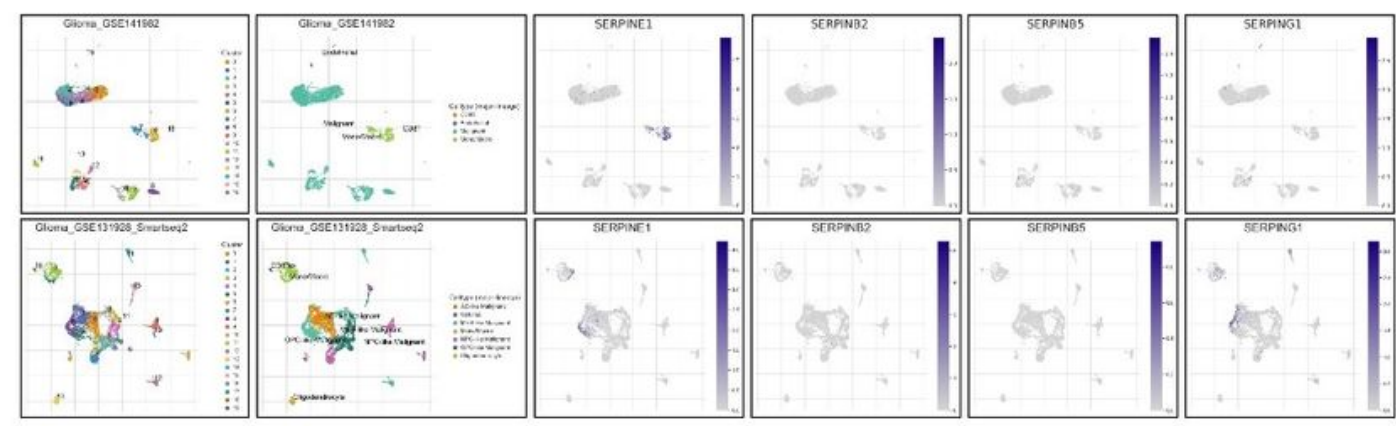

B
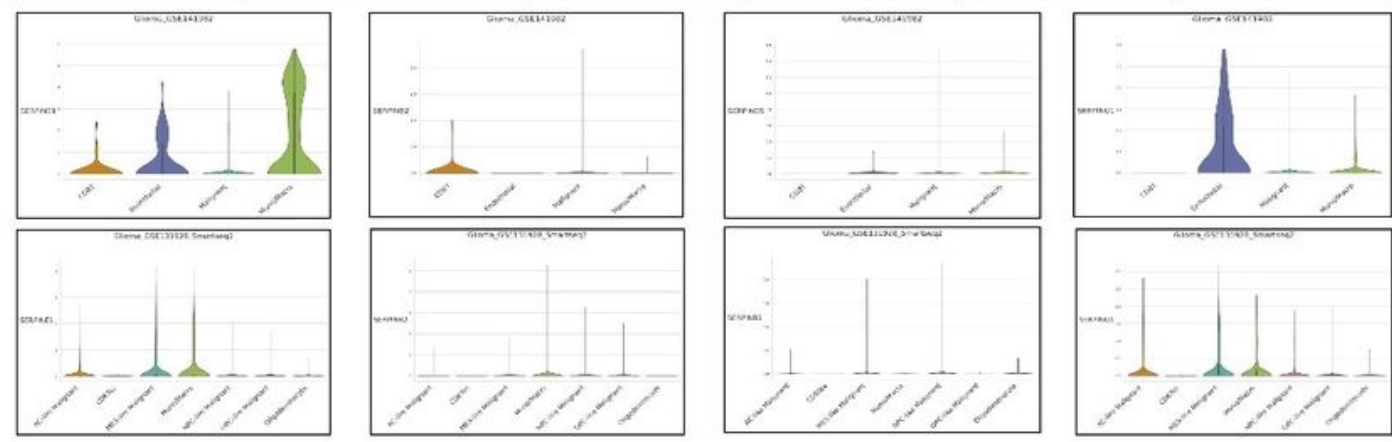

C
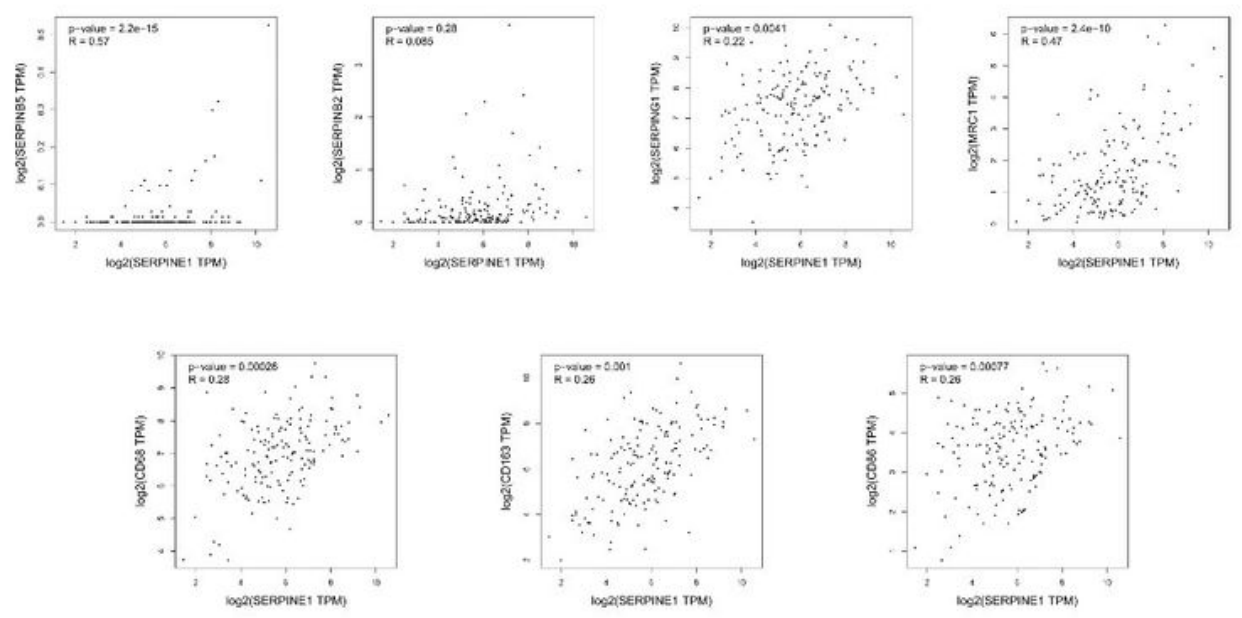

D
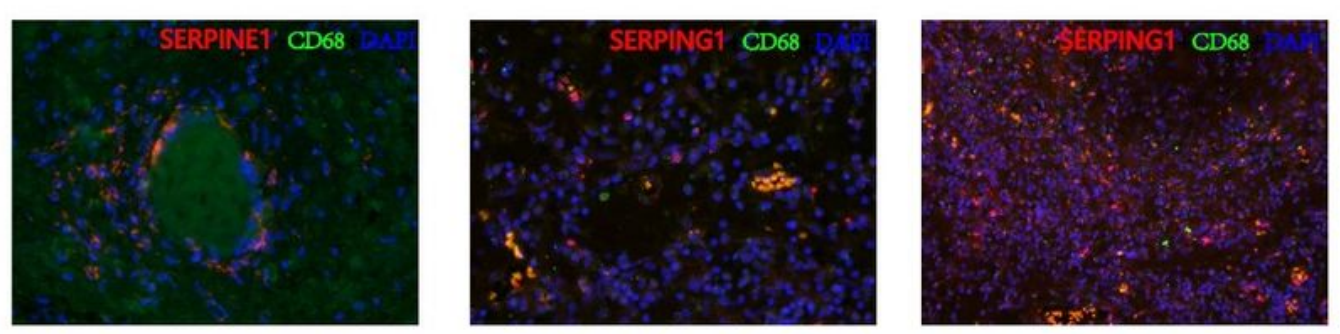

\section{Figure 5}

SERPINs in macrophages might shares similar expression pattern between ACP and GBM. (A) Single cell clustering results display SERPINs expression profiles in GBM. SERPINE1 significantly enriched in malignant cells, macrophages and monocytes, and SERPING1 shows marginally upregulated in macrophages (TISCH). (B) Gene expression level of SERPINS in different cell clusters. SERPINE1 and SERPING1 show evident enrichment (TISCH). (C) Gene correlation results in GBM show SERPINE1 has 
positive correlation with SERPING1 and various markers of polarized macrophages, consisting of CD68, CD86, CD163 and CD206. (D)FISH of paraffin tissue illustrates that SERPINE1 and SERPING1 are found separately co-located in CD68+ macrophages. SERPINE1+ macrophages are more likely located surrounding solid tumor while SERPING1+ in cystic part.

\section{Supplementary Files}

This is a list of supplementary files associated with this preprint. Click to download.

- supplement.docx 